\title{
Development of high yielding two-line hybrid rice in Thailand
}

\author{
Keasinee Tongmark ${ }^{\mathrm{a}}$, Sriprapai Chakhonkaen ${ }^{\mathrm{a}}$, Numphet Sangarwut ${ }^{\mathrm{a}}$, Thiwawan Wasinanon ${ }^{\mathrm{a}}$, \\ Natjaree Panyawut $^{\mathrm{a}}$, Khanittha Ditthab ${ }^{\mathrm{a}}$, Kannika Sikaewtung ${ }^{\mathrm{a}}$, Supaporn Janbuathong ${ }^{\mathrm{b}}$, \\ Suniyom Taprab ${ }^{c}$, Chuanchom Deerusamee ${ }^{\mathrm{d}}$, Amorntip Muangprom ${ }^{\mathrm{a}, *}$ \\ a National Center for Genetic Engineering and Biotechnology, Thailand Science Park, Pathum Thani \\ 12120 Thailand \\ b Pathum Thani Rice Reseach Center, Rice Department, Pathum Thani 12110 Thailand \\ c Rice research and development, Bangkok 10900 Thailand \\ d Chainat Rice Research Center, Rice Department, Chai Nat 17000 Thailand
}

*Corresponding author, e-mail: amorntip.mua@biotec.or.th

Received 15 Oct 2020

Accepted 17 Dec 2020

\begin{abstract}
Thailand is a major producer and exporter of rice. However, the yield per hectare of Thai rice is lower compared with other Southeast Asian countries. We hypothesized that higher-yielding hybrids of Thai varieties could be generated using the temperature-sensitive genic male-sterility (TGMS) two-line hybrid technique. In this study, we evaluated inter-station yield of 14 hybrids generated from two IRRI TGMS rice lines crossed with 13 Thai elite lines/cultivars and planted at different locations in Thailand for two years. One hybrid (Hyb1) showed at least 10\% greater yield than the mean of four high-yielding inbred reference cultivars for all tested years and locations. Eight functional markers controlling agronomically important traits were used to genotype the parents and Hyb1. Positive alleles associated with the important traits were identified among the parents, and four markers were found to be polymorphic. Because contaminants in hybrid seed lots can greatly decrease yield, two of the polymorphic markers were used to assess seed purity of Hyb1. No contaminants were detected, i.e. indicating 100\% seed purity for the hybrid. The results obtained here will be useful for developing high yielding rice cultivars in Thailand.
\end{abstract}

KEYWORDS: two line-hybrid rice, male sterility, functional markers, high quality rice, seed purity, grain yield

\section{INTRODUCTION}

Rice (Oryza sativa L.) is one of the world's most important crops as it is the staple food of more than half of the world's population. With the continual increase of the world's population, decrease in arable land and changing climate, there is an urgent need to increase rice yield to meet the global food demand. Rice is a staple food and major exported product of Thailand. However, the yield per hectare of Thai rice is less than that of other Southeast Asian countries, such as Indonesia, Vietnam, Malaysia, Laos, and Philippines [1]. In order to increase the rice grain yield, the use of hybrid varieties is a promising alternative. Several studies have reported rice hybrids with 15-30\% greater yields than the best inbred varieties [2]. Although the generation of hybrid varieties is laborious, techniques, such as temperature-sensitive genic male-sterility (TGMS) in two-line hybrid system, can facilitate hybrid seed production $[3,4]$. The male sterility or fertility in TGMS lines is controlled by temperature which affects expression of a critical nuclear gene $[5,6]$. Two-line hybrids using the TGMS system overcome several constraints of the threeline hybrid system. Most of the TGMS lines are fertile under low temperature and sterile under high temperature conditions. Thus, TGMS plants can be used as male-sterile lines for hybrid production, or for self-propagation depending on the temperature of culture conditions, which obviates the need for maintainer lines. In addition, the hybrid system using TGMS reduces genetic vulnerability due to the restricted sterile cytoplasm because TGMS genes can be transferred to diverse genetic backgrounds. Furthermore, the TGMS hybrid system allows the use of a wide range of male parent genotypes, eliminating the need for restorer lines and providing not only a broader genetic base of the hybrids, but also more choices for developing hybrids with desirable traits [5].

The success of hybrid technology depends 
mainly on the production of genetically pure hybrid seeds to ensure heterosis. It has been reported that $1 \%$ impurity in the hybrid seed reduces yield of hybrid by about $100 \mathrm{~kg} / \mathrm{ha}$ [7]. Therefore, it is critical to monitor genetic purity of parental lines at each stage of hybrid seed production.

Genetic purity of hybrid seeds depends on the purity of the parental lines and the processes for hybrid seed production, including the presence of pollen shedders and physical admixtures [8]. Seed purity is conventionally determined by Grow-OutTest (GOT). GOT is based on growing representative samples of target seeds and observing their phenotypes. Because the test is based on morphological traits, which can be significantly affected by environments, accurate determination of seed purity is difficult. In addition, many modern cultivars are phenotypically less distinct from one another, making morphological evaluation more challenging. Besides, GOT is time-consuming, tedious, costly, and requires excessive use of land $[9,10]$. Alternatively, seed purity can be assessed much more easily using molecular markers based on DNA sequence variation.

Simple sequence repeat (SSR) marker provides an unbiased means of crop identification using DNA sequence variation. SSR markers are the most widely used for seed purity tests and cultivar identification because of their abundance, uniform distribution and co-dominant status [11-13]. Several studies have reported the use of SSR markers for cultivar identification and seed purity assessment in several crops including rice [12], sunflower [14], maize [15], wheat [16], and pigeon pea [13]. Importantly, the use of SSR markers for seed purity test has been reported by many groups to be comparable with GOT $[8,15,17]$.

Functional markers are DNA markers developed from polymorphic sites located in genes that cause phenotypic trait variation [18]. Functional markers are directly linked to the allele of the traits of interest [19]. Therefore, functional markers are superior to DNA markers with no particular functional association such as SSRs, especially in marker-assisted breeding. Several genes controlling agronomically desirable traits, such as fragrance, high eating and cooking quality, high yield, and resistance to biotic and abiotic stress, have been reported and functional markers for these genes have been developed [20-26]. Rice lines harboring functional markers for these traits (positive alleles) will be useful for rice breeding programs. In addition, polymorphism of functional markers among
Table 1 Hybrids and their parents.

\begin{tabular}{lll}
\hline No & Hybrid & Parent \\
\hline 1 & Hyb1 & IR68301-11-6-4-4-3-6-6xPTT1 \\
2 & Hyb2 & IR76761-4-3-17-34-35xCK81 \\
3 & Hyb3 & IR76761-4-3-17-34-35xKLG96006 \\
4 & Hyb4 & IR76761-4-3-17-34-35xRD31 \\
5 & Hyb5 & IR76761-4-3-17-34-35xSPR93014 \\
6 & Hyb6 & IR76761-4-3-17-34-35xKLG02008 \\
7 & Hyb7 & IR76761-4-3-17-34-35xSPR93039-PTT \\
8 & Hyb8 & IR76761-4-3-17-34-35xPTT98044 \\
9 & Hyb9 & IR76761-4-3-17-34-35xSPR90 \\
10 & Hyb10 & IR76761-4-3-17-34-35xSPR1 \\
11 & Hyb11 & IR76761-4-3-17-34-35xSPR3 \\
12 & Hyb12 & IR76761-4-3-17-34-35xB11 \\
13 & Hyb13 & IR76761-4-3-17-34-35xPTT1 \\
14 & Hyb14 & IR76761-4-3-17-34-35xPSL60-2 \\
\hline
\end{tabular}

cultivars can be used for seed purity test.

This study aimed to evaluate the yield of twoline hybrids, to identify positive alleles for agronomically desirable traits and polymorphic functional markers between parents of high yielding hybrids and to test the seed purity of selected high-yielding hybrids. The obtained information will be useful for future rice breeding programs and for two-line hybrid rice development in Thailand.

\section{MATERIALS AND METHODS}

\section{Plant materials}

Two TGMS rice lines (IR68301-11-6-4-4-3-6-6 and IR76761-4-3-17-34-35) obtained from the International Rice Research Institute (IRRI) and 13 Thai elite lines/cultivars, obtained from the Rice Department, Ministry of Agriculture and Cooperatives, Bangkok, were used for generating hybrids (Table 1). Several hybrids were generated and used for preliminary yield evaluations, and the promising high-yielding hybrids were selected for intra-station yield evaluations. Subsequently, the 14 selected high-yielding hybrids were used for inter-station yield evaluations for two years, at two locations for each year.

For molecular analysis, one selected highyielding hybrid (Hyb1) and the parents (B2 and PTT1) were tested. B11 was used as a positive control for TGW6 [25], Ghd7-05SNP [25] and Gn1a17SNP [25]. B80 was used as a positive control for S5n [22], Wx [24], Wx-Glu [26], and SSIIa [20]. B72 was used as a positive control for Badh2 [21], and was used as a negative control for TGW6 [25], Ghd7-05SNP [25] and Gn1a-17SNP [25]. RD31 was used as a negative control for $S 5 n$ [22], $W x$ [24], 
Wx-Glu [26], SSIIa [20], and Badh2 [21].

\section{Experimental design}

Field experiments were conducted in dry seasons in 2015 and 2016 at two locations for each year, using a randomized complete block design with three replications. In 2015, the experiments were conducted at the Pathum Thani Rice Research Center (PTrc) and the National Center for Genetic Engineering and Biotechnology (BIOTEC), and in 2016 at the PTrc and the Chainat Rice Research Center (CNrc). The 14 hybrids and 4 high-yielding inbred Thai reference cultivars (RD31, PSL2, PTT1, and RD47) were transplanted one seedling per hill, at a hill spacing of $20.0 \times 20.0 \mathrm{~cm}$ at PTrc and CNrc, and at a hill spacing of $25.0 \times 25.0 \mathrm{~cm}$ at BIOTEC. For each replication, six rows, with 26 plants per row $(6 \times 26$ plants), were planted for each accession. Harvesting areas were $4 \times 24$ plants inside each plot. Nitrogen was applied as urea $(112.5 \mathrm{~kg} / \mathrm{ha})$ in two splits: $37.5 \mathrm{~kg} / \mathrm{ha}$ basally and $112.5 \mathrm{~kg} / \mathrm{ha} 50$ days after transplanting. Phosphate and potassium were applied basally as superphosphate $(37.5 \mathrm{~kg} / \mathrm{ha})$ and potassium chloride $(37.5 \mathrm{~kg} / \mathrm{ha})$, respectively. Crop management followed the standard cultural practices. Fields were kept flooded at 5-10 cm depth until one week after flowering, when surface water was drained off.

\section{Sampling and measurements}

Time to flowering was recorded when $75 \%$ of plants in each plot were flowering. Grain yields were determined from 96 plants $(4 \times 24)$ inside each plot and adjusted to standard moisture content of $0.14 \mathrm{~g} \mathrm{H}_{2} \mathrm{O} / \mathrm{g}$. Agronomic traits, including number of spikelets per panicle, spikelet fertility (100 $\times$ filled spikelet number/total spikelet number, $\%)$ and 1000-grain weight, were investigated and determined from random ten plants of each plot. Numbers of spikelets per panicle and spikelet fertility were determined using one panicle per plant. Numbers of panicles per plant were determined from active tillers of each plant from the random sampling of ten plants. Plant height was measured from the plant base to the tip of the highest leaf or panicle from the ten plants.

Data were analyzed using analysis of variance (ANOVA) [27], and means of varieties were compared based on the least significant difference test (LSD) at the 0.05 probability level for each location and year.

\section{Molecular analysis}

Genomic DNA was isolated from fresh leaves using the Cethyl-trimethylammonium bromide (CTAB) method [28]. For identification of positive alleles for agronomically desirable traits and polymorphic markers, pooled DNA from ten individuals of each parent and pooled DNA from 30 individual plants of Hyb1 were tested along with positive and negative DNA samples. For seed purity test, 30 random plants of Hyb1 were tested individually along with pooled DNA samples of each parent (ten plants, each). Details of the tested primers are shown in Table S1. TGW6, Ghd7 and Gn1a are genes associated with yield [25]. Badh2 is a gene affecting fragrance [21]. $W x$ is a gene influencing amylose content [24] and $W x$-Glu is a functional marker used to distinguish glutinous rice from normal rice [26]. SSIIa is a gene associated with gelatinization temperature [20]. Badh2, Wx and SSIIa are genes controlling eating and cooking quality in rice. $S 5 n$ is associated with spikelet fertility of an indicajaponica hybrid [22]. PCR experiments were performed as previously described [29]. The resulting PCR products were electrophoretically separated in $2 \%$ agarose gel. DNA patterns were observed by ethidium bromide staining.

\section{RESULTS}

\section{Yield and yield components}

Due to high variation across all locations and years, the yield and yield components were analysed separately by locations and years. In 2015, the 14 hybrids and 4 high-yielding inbred reference cultivars widely cultivated in Thailand (RD31, PSL2, PTT1, and RD47) were planted at PTrc and BIOTEC, one seedling per hill, at a hill spacing of $20.0 \mathrm{~cm} \times 20.0 \mathrm{~cm}$ at PTrc and $25.0 \mathrm{~cm} \times 25.0 \mathrm{~cm}$ at BIOTEC. At PTrc (Table 2), the 4 high-yielding inbred reference cultivars, RD31, PSL2, PTT1, and RD47, produced grain yields of $6.6,6.6,6.3$, and $6.9 \mathrm{t} / \mathrm{ha}$, respectively, with an average of $6.6 \mathrm{t} / \mathrm{ha}$. The hybrids produced grain yields in a range of 6.0-7.7 t/ha, with an average of 7.2 t/ha. Hyb1 produced the highest yield, $7.7 \mathrm{t} / \mathrm{ha}$, which was $16 \%$ higher than the mean check (mean of the four reference cultivars). Although the overall difference in grain yield between the high-yielding inbred reference cultivars and the hybrids was statistically insignificant, there were 7 hybrids showing 12$16 \%$ higher yield than the mean check. Details of yields and yield components of the hybrids and the 
Table 2 Inter-station yield evaluation of two-line hybrids planted at the Pathum Thani Rice Research Center (PTrc) in 2015.

\begin{tabular}{|c|c|c|c|c|c|c|c|c|c|}
\hline No & List & $\begin{array}{l}\text { Gain yield } \\
\text { (t/ha) }\end{array}$ & $\begin{array}{l}\% \text { of Mean } \\
\text { check }(6.6)\end{array}$ & $\begin{array}{c}\text { Spikelet } \\
\text { fetility (\%) }\end{array}$ & $\begin{array}{l}\text { No. of seeds } \\
\text { per penicle }\end{array}$ & $\begin{array}{l}1000 \text { grain } \\
\text { weight }(g)\end{array}$ & $\begin{array}{l}\text { No. of } \\
\text { tillers }\end{array}$ & $\begin{array}{c}\text { Flowering } \\
\text { time (d) }\end{array}$ & $\begin{array}{l}\text { Height } \\
\text { (cm) }\end{array}$ \\
\hline 1 & Hyb1 & $7.7 \pm 0.85^{\mathrm{ab}}$ & 116.0 & $82.0 \pm 2.04^{\mathrm{abc}}$ & $139.5 \pm 26.63^{\mathrm{bcd}}$ & $27.5 \pm 0.52^{\mathrm{ab}}$ & $11.5 \pm 0.81^{a}$ & $90.0 \pm 4.36^{a}$ & $\overline{136.7 \pm 7.57^{\mathrm{a}}-\mathrm{d}}$ \\
\hline 2 & Hyb2 & $7.1 \pm 1.52^{\mathrm{abc}}$ & 107.4 & $84.3 \pm 2.89^{\mathrm{ab}}$ & $188.8 \pm 2.27^{\mathrm{a}}$ & $24.7 \pm 0.33^{\mathrm{ef}}$ & $12.0 \pm 3.70^{\mathrm{a}}$ & $80.5 \pm 0.71^{e f}$ & $129.8 \pm 1.50^{\mathrm{b}-\mathrm{f}}$ \\
\hline 3 & Hyb3 & $7.4 \pm 0.66^{\mathrm{abc}}$ & 111.9 & $73.0 \pm 1.68^{\mathrm{bcd}}$ & $163.7 \pm 13.3^{a b c}$ & $26.1 \pm 0.16^{b-f}$ & $13.2 \pm 3.12^{\mathrm{a}}$ & $85.0 \pm 2.65^{b c d}$ & $131.4 \pm 3.41^{\mathrm{b}-\mathrm{f}}$ \\
\hline 4 & Hyb4 & $7.5 \pm 0.63^{\mathrm{ab}}$ & 114.2 & $83.7 \pm 0.68^{a b c}$ & $153.6 \pm 6.43^{a-d}$ & $25.6 \pm 0.39^{c-f}$ & $11.7 \pm 2.14^{\mathrm{a}}$ & $74.7 \pm 0.58^{g h}$ & $131.0 \pm 2.69^{b-f}$ \\
\hline 5 & Hyb5 & $7.4 \pm 2.02^{\mathrm{ab}}$ & 112.6 & $81.0 \pm 6.88^{\mathrm{a}-\mathrm{d}}$ & $150.5 \pm 12.49^{\mathrm{a}-\mathrm{d}}$ & $28.3 \pm 1.80^{\mathrm{a}}$ & $13.3 \pm 0.64^{a}$ & $78.0 \pm 1.73^{\text {fg }}$ & $138.2 \pm 1.74^{\mathrm{ab}}$ \\
\hline 6 & Hyb6 & $7.6 \pm 0.56^{\mathrm{ab}}$ & 115.7 & $84.0 \pm 1.97^{\mathrm{ab}}$ & $149.4 \pm 15.86^{\mathrm{bcd}}$ & $26.6 \pm 0.20^{\mathrm{a}-\mathrm{d}}$ & $14.7 \pm 1.21^{\mathrm{a}}$ & $73.0 \pm 0.00^{g h}$ & $137.9 \pm 5.89^{\mathrm{ab}}$ \\
\hline 7 & Hyb7 & $7.1 \pm 0.78^{\mathrm{abc}}$ & 107.6 & $86.3 \pm 0.95^{\mathrm{a}}$ & $135.8 \pm 16.26^{\mathrm{bcd}}$ & $25.7 \pm 0.17^{\mathrm{c}-\mathrm{f}}$ & $14.6 \pm 0.35^{a}$ & $73.5 \pm 0.71^{\mathrm{gh}}$ & $130.6 \pm 2.27^{\mathrm{b}-\mathrm{f}}$ \\
\hline 8 & Hyb8 & $7.5 \pm 1.15^{\mathrm{ab}}$ & 114.1 & $84.0 \pm 1.73^{\mathrm{ab}}$ & $145.3 \pm 7.40^{\mathrm{bcd}}$ & $25.4 \pm 0.53^{c-f}$ & $13.3 \pm 1.03^{\mathrm{a}}$ & $73.7 \pm 0.58^{g h}$ & $134.1 \pm 4.35^{\mathrm{a}-\mathrm{e}}$ \\
\hline 9 & Hyb9 & $6.0 \pm 2.86^{\mathrm{bc}}$ & 90.2 & $72.7 \pm 9.11^{\mathrm{bcd}}$ & $162.6 \pm 3.21^{a b c}$ & $24.8 \pm 1.94^{\mathrm{def}}$ & $14.1 \pm 2.41^{\mathrm{a}}$ & $75.3 \pm 3.06^{g h}$ & $133.9 \pm 6.46^{\mathrm{a}-\mathrm{e}}$ \\
\hline 10 & Hyb10 & $7.2 \pm 1.13^{\mathrm{abc}}$ & 108.7 & $85.3 \pm 4.15^{\mathrm{ab}}$ & $160.2 \pm 10.19^{a b c}$ & $24.4 \pm 1.52^{\mathrm{f}}$ & $13.8 \pm 1.22^{\mathrm{a}}$ & $76.0 \pm 3.61^{g}$ & $137.2 \pm 1.70^{\mathrm{abc}}$ \\
\hline 11 & Hyb11 & $6.0 \pm 0.78^{b c}$ & 90.8 & $71.0 \pm 1.24^{\mathrm{cd}}$ & $131.5 \pm 15.16^{\mathrm{cd}}$ & $25.3 \pm 0.20^{\mathrm{c}-\mathrm{f}}$ & $13.1 \pm 2.41^{\mathrm{a}}$ & $86.0 \pm 0.00^{b c}$ & $138.4 \pm 3.81^{\mathrm{ab}}$ \\
\hline 12 & Hyb12 & $7.6 \pm$ & 114.5 & $82.3 \pm 2.60^{\mathrm{abc}}$ & $147.8 \pm 17.14^{\mathrm{bcd}}$ & $0.50^{\mathrm{c}-\mathrm{f}}$ & $13.1 \pm 1.92^{\mathrm{a}}$ & ND & $131.0 \pm 2.78^{b-f}$ \\
\hline 13 & Hyb13 & $73^{a b c}$ & 107.7 & $84.7=$ & $134.4 \pm 4.50^{\mathrm{bcd}}$ & $25.0 \pm 0.21^{\mathrm{def}}$ & $14.2 \pm 1.71^{\mathrm{a}}$ & $74.7 \pm 2.89^{g h}$ & $127.9 \pm 7.00^{c-h}$ \\
\hline 14 & Hyb14 & $7.2 \pm 0.90^{\mathrm{abc}}$ & 109.1 & $82.3 \pm 1.17^{\mathrm{abc}}$ & $172.0 \pm 16.76^{\mathrm{ab}}$ & $24.7 \pm 0.07^{\mathrm{ef}}$ & $11.5 \pm 3.90^{\mathrm{a}}$ & ND & $133.5 \pm 5.52^{\mathrm{a}-\mathrm{e}}$ \\
\hline 15 & RD31 & $.60^{a b c}$ & & $0.64^{\mathrm{a}-\mathrm{d}}$ & 14 & $28^{\mathrm{b}-\mathrm{e}}$ & 13.2 & $58^{\mathrm{b}}$ & $.01^{\mathrm{a}}$ \\
\hline 16 & PSL2 & $6.6 \pm 0.71^{\mathrm{abc}}$ & Mean & $80.0 \pm 3.18^{\mathrm{a}-\mathrm{d}}$ & $146.0 \pm 18.76^{\mathrm{bc}}$ & $28.3 \pm 0.25^{\mathrm{a}}$ & $11.3 \pm 0.76^{\mathrm{a}}$ & $86.3 \pm 4.73^{b}$ & $120.3 \pm$ \\
\hline 17 & PTT1 & $6.3 \pm 0.98^{b c}$ & check & $81.0 \pm 1.14^{\mathrm{a}-\mathrm{d}}$ & $118.5 \pm 19.89^{d}$ & $26.9 \pm 0.23^{\mathrm{abc}}$ & $13.1 \pm 0.92^{\mathrm{a}}$ & $90.0 \pm 1.73^{\mathrm{a}}$ & $132.1 \pm 5.52^{\mathrm{d}-\mathrm{f}}$ \\
\hline 18 & RD47 & $6.9 \pm 0.86^{a b c}$ & & $69.0 \pm 1.26^{\mathrm{d}}$ & $146.1 \pm 24.43^{b c}$ & $28.1 \pm 0.73^{a}$ & $11.9 \pm 0.81^{\mathrm{a}}$ & $84.3 \pm 1.53^{b c}$ & $122.8 \pm 8.40^{\text {fgh }}$ \\
\hline
\end{tabular}

Within each column, means followed by a common letter are not significantly different by the LSD test at the $5 \%$ level of significance. ND means not determined. Lot numbers 1-14 represent hybrids from crosses of TGMS lines with Thai cultivars (see Table 1). Lot numbers 15-18 represent high-yielding inbred Thai reference cultivars for comparison. Mean check is the mean grain yield of the four reference cultivars.

high-yielding reference cultivars were also shown in Table 2. Most of the hybrids, heights were similar to PTT1, but shorter than RD31 and taller than PSL2 and RD47. For flowering time, most of the hybrids, except Hyb1, flowered earlier than most of the reference cultivars. Hyb1 showed later flowering (90 days), which was similar to that of PTT1.

At BIOTEC (Table 3), the 4 high-yielding inbred reference cultivars, RD31, PSL2, PTT1, and RD47, produced grain yields of $10.2,8.4,9.4$, and $8.7 \mathrm{t} / \mathrm{ha}$, respectively, with an average of $9.2 \mathrm{t} / \mathrm{ha}$. The hybrids produced grain yields in a range of 7.5$12.6 \mathrm{t} / \mathrm{ha}$, with an average of $10.0 \mathrm{t} / \mathrm{ha}$. Hyb1 produced the highest yield, $12.6 \mathrm{t} / \mathrm{ha}$, which was $37 \%$ higher than the mean check. There were 6 hybrids showing 15-37\% higher yield than the mean check. Details of yields and yield components of the hybrids and the high-yielding inbred reference cultivars were also shown in Table 3 . For plant height, most of the hybrids were similar to PTT1, but shorter than RD31 and taller than PSL2 and RD47. Hyb1 was as tall as RD31. For flowering time, most of the hybrids except Hyb1 flowered earlier than most of the reference cultivars. Hyb1 showed later flowering (100 days) compared to other varieties.

In 2016, the 14 hybrids and 4 high-yielding inbred reference cultivars were planted at PTrc and CNrc, one seedling per hill, at a hill spacing of $20.0 \mathrm{~cm} \times 20.0 \mathrm{~cm}$. At PTrc (Table 4), the 4 highyielding inbred reference cultivars, RD31, PSL2,
PTT, and RD47 produced grain yields of 4.56, 3.7, 3.3 , and $3.8 \mathrm{t} / \mathrm{ha}$, respectively, with an average of $3.8 \mathrm{t} / \mathrm{ha}$. The hybrids produced grain yields in a range of $2.7-5.4 \mathrm{t} / \mathrm{ha}$, with an average of $4.3 \mathrm{t} / \mathrm{ha}$. There were 7 hybrids producing $15-41 \%$ higher yield than the mean check. Details of yields and yield components of the hybrids and the highyielding inbred reference cultivars were also shown in Table 4. For plant height, most of the hybrids were taller than most of the high-yielding inbred reference cultivars, except RD31. For flowering time, most of the hybrids, except Hyb1, flowered earlier than most of the reference cultivars. Hyb1 showed later flowering (119 days) compared to other varieties.

At CNrc (Table 5), the 4 high-yielding inbred reference cultivars, RD31, PSL2, PTT, and RD47 produced grain yields of 5.3, 5.2, 4.9, and 5.9 t/ha, respectively, with an average of $5.3 \mathrm{t} / \mathrm{ha}$. The hybrids produced grain yields in a range of 4.2$8.8 \mathrm{t} / \mathrm{ha}$, with an average of $6.3 \mathrm{t} / \mathrm{ha}$. There were 5 hybrids showing 10-66.5\% higher yield than the mean check. Details of yields and yield components of the hybrids and the high-yielding inbred reference cultivars were also shown in Table 5. For plant height, most of the hybrids were taller than PSL2, but all of the hybrids were shorter than RD31. For flowering time, most of the hybrids, except Hyb1, flowered earlier than most of the reference cultivars. Hyb1 showed later flowering similar to PTT1. 
Table 3 Inter-station yield evaluation of two-line hybrids planted at the National Center for Genetic Engineering and Biotechnology (BIOTEC) in 2015.

\begin{tabular}{|c|c|c|c|c|c|c|c|c|c|}
\hline No & List & $\begin{array}{l}\text { Gain yield } \\
\text { (t/ha) }\end{array}$ & $\begin{array}{l}\% \text { of Mean } \\
\text { check }(9.2)\end{array}$ & $\begin{array}{c}\text { Spikelet } \\
\text { fetility (\%) }\end{array}$ & $\begin{array}{l}\text { No. of seeds } \\
\text { per penicle }\end{array}$ & $\begin{array}{l}1000 \text { grain } \\
\text { weight }(\mathrm{g})\end{array}$ & $\begin{array}{l}\text { No. of } \\
\text { tillers }\end{array}$ & $\begin{array}{l}\text { Time to } \\
\text { flower (d) }\end{array}$ & $\begin{array}{l}\text { Height } \\
\text { (cm) }\end{array}$ \\
\hline 1 & Hyb1 & $12.6 \pm 4.15^{\mathrm{a}}$ & 7.4 & $89.7 \pm 1.02^{\mathrm{ab}}$ & 19 & 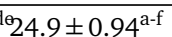 & $.7 \pm 4.51^{b c}$ & $100.7 \pm 4.51^{\mathrm{a}}$ & $128.0 \pm 1.68^{\mathrm{ab}}$ \\
\hline $\begin{array}{l}1 \\
2\end{array}$ & Hyb2 & $9.7 \pm 1.74^{\mathrm{abc}}$ & 106.0 & $88.7 \pm 4.77^{\mathrm{abc}}$ & $250.9 \pm 15.44^{\mathrm{a}}$ & $58^{g}$ & $22.7 \pm 3.79^{a b c}$ & $82.3 \pm 2.89^{\mathrm{e}-\mathrm{h}}$ & $114.7 \pm 2.34^{\mathrm{d}-\mathrm{h}}$ \\
\hline 3 & Hyb3 & $12.5 \pm 3.33^{\mathrm{a}}$ & 136.7 & $86.7 \pm 3.04^{\mathrm{a}-\mathrm{d}}$ & $205.5 \pm 19.90^{b}$ & $54^{d-g}$ & $27.0 \pm 11.36^{\mathrm{abc}}$ & $88.0 \pm 5.29^{\text {cde }}$ & $112.7 \pm 2.42^{\mathrm{e}-\mathrm{i}}$ \\
\hline 4 & Hyb4 & $11.6 \pm 1.89^{\mathrm{ab}}$ & 126.2 & $87.7 \pm 3.14^{\mathrm{a}-\mathrm{d}}$ & $193.4 \pm 22.67^{c}$ & $23.9 \pm 1.31^{\mathrm{b}-\mathrm{g}}$ & $27.0 \pm 4.36^{\mathrm{abc}}$ & $78.3 \pm 0.58^{g h}$ & $119.7 \pm 0.53^{\text {de }}$ \\
\hline 5 & Hyb5 & $11.6 \pm 2.47^{\mathrm{ab}}$ & 126.8 & $88.0 \pm 3.04^{\mathrm{abc}}$ & $202.6 \pm 34.65^{c}$ & $25.2 \pm 1.92^{\mathrm{a}-\mathrm{d}}$ & $25.7 \pm 3.21^{\mathrm{abc}}$ & $79.0 \pm 1.00^{\mathrm{gh}}$ & $118.3 \pm 3.51^{\mathrm{def}}$ \\
\hline 6 & Hyb6 & $11.7 \pm 2.19^{\mathrm{ab}}$ & 127.7 & $89.3 \pm 1.04^{\mathrm{ab}}$ & $190.3 \pm 12.07^{c}$ & $25.1 \pm 0.69^{\mathrm{a}-\mathrm{e}}$ & $21.3 \pm 6.51^{a b c}$ & $79.0 \pm 1.00^{g h}$ & $121.0 \pm 0.76^{b c d}$ \\
\hline 7 & Hyb7 & $8.1 \pm 1.16^{\mathrm{cd}}$ & 88.6 & $80.3 \pm 7.26^{\mathrm{b}-\mathrm{f}}$ & $176.4 \pm 21.63^{\mathrm{e}}$ & $24.3 \pm 0.72^{b-g}$ & $22.3 \pm 1.53^{a b c}$ & $77.7 \pm 2.08^{h}$ & $113.3 \pm 4.82^{\mathrm{d}-\mathrm{h}}$ \\
\hline 8 & Hyb8 & $7.8 \pm 0.86^{\mathrm{cd}}$ & 85.0 & $87.7 \pm 1.45^{\mathrm{a}-\mathrm{d}}$ & $197.5 \pm 39.50^{c}$ & $23.3 \pm 0.40^{c-g}$ & $26.0 \pm 1.73^{a b c}$ & $81.3 \pm 2.08^{\text {fgh }}$ & $119.3 \pm 4.66^{\mathrm{def}}$ \\
\hline 9 & Hyb9 & $10.6 \pm 2.17^{\mathrm{abc}}$ & 115.3 & $86.0 \pm 5.02^{\mathrm{a}-\mathrm{d}}$ & $235.6 \pm 21.52^{\mathrm{a}}$ & $25.4 \pm 1.70^{\mathrm{abc}}$ & $21.3 \pm 5.51^{\mathrm{abc}}$ & $80.3 \pm 2.08^{\text {fgh }}$ & $118.0 \pm 2.99^{\mathrm{d}-\mathrm{g}}$ \\
\hline 10 & Hyb10 & $8.9 \pm 3.99^{\mathrm{bcd}}$ & 97.3 & $86.3 \pm 1.77^{\mathrm{a}-\mathrm{d}}$ & $184.3 \pm 6.36^{\mathrm{de}}$ & $22.9 \pm 0.39^{\mathrm{d}-\mathrm{g}}$ & $28.0 \pm 5.57^{\mathrm{ab}}$ & $78.0 \pm 1.00^{h}$ & $117.3 \pm 0.61^{\mathrm{d}-\mathrm{g}}$ \\
\hline 11 & Hyb11 & $9.4 \pm 1.91^{a b c}$ & 102.7 & $81.0 \pm 6.85^{\mathrm{a}-\mathrm{f}}$ & $210.0 \pm 38.52^{b}$ & $23.9 \pm 0.89^{b-g}$ & $24.3 \pm 6.81^{a b c}$ & $92.3 \pm 7.77^{\mathrm{bc}}$ & $121.3 \pm 5.40^{\mathrm{a}-\mathrm{d}}$ \\
\hline 12 & Hyb12 & $9.0 \pm 1.30^{\mathrm{bcd}}$ & 98.5 & $82.0 \pm 11.33^{\mathrm{a}-}$ & $181.3 \pm 20.03^{\mathrm{d}}$ & $24.5=$ & $27.3 \pm 2.52^{\mathrm{ab}}$ & $78.0 \pm 2.00^{h}$ & $114.7 \pm 4.46^{\mathrm{d}-\mathrm{h}}$ \\
\hline 13 & Hyb13 & $9.0 \pm 1.32^{\mathrm{bcd}}$ & 97.7 & $90.7 \pm 3.39^{\mathrm{a}}$ & $169.7=$ & $22.6 \pm 0.79^{f g}$ & $24.0 \pm 3.00^{\mathrm{abc}}$ & $77.7 \pm 1.53^{h}$ & $110.0 \pm 2.00^{\text {ghi }}$ \\
\hline 14 & Hyb14 & $7.5 \pm 1.61^{\mathrm{cd}}$ & 82.1 & $86.0 \pm 6.03^{\mathrm{a}-\mathrm{d}}$ & $201.3 \pm 16.00^{c}$ & $23.2 \pm 0.64^{c-g}$ & $18.3 \pm 3.21^{c}$ & $77.0 \pm 1.00^{h}$ & $127.3 \pm 8.52^{a b c}$ \\
\hline 15 & D31 & $13^{\mathrm{abc}}$ & & $29^{a-d}$ & 181 & $28^{\mathrm{ab}}$ & $29.0 \pm 5.57^{a}$ & $52^{\mathrm{ab}}$ & 128.7 \\
\hline 16 & PSL2 & $8.4 \pm 2.98^{\mathrm{bcd}}$ & & $82.7 \pm 4.55^{\mathrm{a}-\mathrm{e}}$ & $167.7 \pm 21.19^{\mathrm{e}}$ & $.60^{\mathrm{b}-\mathrm{g}}$ & $26.0 \pm 8.19^{\mathrm{abc}}$ & $91.7 \pm 4.51^{\mathrm{bc}}$ & $\begin{array}{r}120.1 \\
97.3\end{array}$ \\
\hline 17 & PTT1 & $9.3 \pm 2.39^{\mathrm{abc}}$ & & $79.0 \pm 6.87^{\mathrm{c}-\mathrm{f}}$ & $171.1 \pm 42.79^{\mathrm{e}}$ & $23.4 \pm 0.58^{\mathrm{c}-\mathrm{g}}$ & $23.7 \pm 2.08^{a b c}$ & $91.3 \pm 3.21^{\mathrm{bcd}}$ & $114.0 \pm 7.21^{\mathrm{d}-\mathrm{h}}$ \\
\hline 18 & RD47 & $8.7 \pm 2.20^{b c d}$ & 12) & $77.7 \pm 3.71^{\text {edf }}$ & $164.3 \pm 31.53^{\mathrm{e}}$ & $26.8 \pm 0.05^{\mathrm{a}}$ & $20.0 \pm 4.58^{b c}$ & $85.0 \pm 1.00^{\mathrm{d}-\mathrm{g}}$ & $107.0 \pm 4.50^{h i}$ \\
\hline
\end{tabular}

Within each column, means followed by a common letter are not significantly different by the LSD test at the $5 \%$ level of significance. Lot numbers 1-14 represent hybrids from crosses of TGMS lines with Thai cultivars (see Table 1). Lot numbers 15-18 represent high-yielding inbred Thai reference cultivars for comparison. Mean check is the mean grain yield of the four reference cultivars.

Table 4 Inter-station yield evaluation of two-line hybrids planted at the Pathum Thani Rice Research Center (PTrc) in 2016.

\begin{tabular}{|c|c|c|c|c|c|c|c|c|c|}
\hline No & List & $\begin{array}{l}\text { Gain yield } \\
\text { (t/ha) }\end{array}$ & $\begin{array}{l}\% \text { of Mean } \\
\text { check (3.8) }\end{array}$ & $\begin{array}{c}\text { Spikelet } \\
\text { fetility (\%) }\end{array}$ & $\begin{array}{l}\text { No. of seeds } \\
\text { per penicle }\end{array}$ & $\begin{array}{l}1000 \text { grain } \\
\text { weight }(\mathrm{g})\end{array}$ & $\begin{array}{l}\text { No. of } \\
\text { tillers }\end{array}$ & $\begin{array}{l}\text { Time to } \\
\text { flower (d) }\end{array}$ & $\begin{array}{l}\text { Height } \\
(\mathrm{cm})\end{array}$ \\
\hline 1 & Hyb1 & $4.2 \pm 0.68^{\mathrm{a}-\mathrm{b}}$ & 110.3 & $76.0 \pm 1.34^{\mathrm{a}-\mathrm{e}}$ & $148.0 \pm 16.71^{\mathrm{a}}$ & $26.6 \pm 0.05^{a b c}$ & $8.0 \pm 0.70^{\mathrm{b}}$ & $119.0 \pm 1.15^{\mathrm{a}}$ & $127.9 \pm 5.90^{\mathrm{b}} \mathrm{cc}$ \\
\hline 2 & Hyb2 & $\pm 0.57^{\mathrm{ab}}$ & 118.3 & $74.7 \pm 3.24^{\mathrm{a}-\mathrm{f}}$ & $154.3 \pm 15.80^{\mathrm{ab}}$ & c $23.6 \pm 1.17^{\mathrm{i}}$ & $10.0 \pm 1.70^{\mathrm{ab}}$ & $98.0 \pm 0.58^{\mathrm{e}}$ & $116.5 \pm 8.08^{f-i}$ \\
\hline 3 & Hyb3 & $=0.19^{\mathrm{e}}$ & 70.5 & $0.3 \pm 7.86^{\mathrm{c}-\mathrm{f}}$ & $169.3 \pm 6.00^{\mathrm{ab}}$ & $25.4 \pm 0.45^{\mathrm{c}-\mathrm{g}}$ & $8.0 \pm 1.10^{\mathrm{b}}$ & $118.0 \pm 1.53^{\mathrm{a}}$ & $128.2 \pm 6.49^{\mathrm{bcc}}$ \\
\hline 4 & Hyb4 & $4.0 \pm 0.61^{\mathrm{bcd}}$ & 105.6 & $65.0 \pm 5.13^{\mathrm{f}}$ & $135.0 \pm 20.56^{\mathrm{cd}}$ & $25.0 \pm 0.27^{\mathrm{d}-\mathrm{h}}$ & $10.0 \pm 2.36^{\mathrm{ab}}$ & $98.0 \pm 1.00^{\mathrm{e}}$ & $122.9 \pm 7.49^{\mathrm{c}-\mathrm{h}}$ \\
\hline 5 & Hyb5 & $5 \pm 0.14^{\mathrm{ab}}$ & 117.5 & $66.7 \pm 2.18^{\mathrm{ef}}$ & $144.0 \pm 13.86^{\mathrm{bcd}}$ & ${ }^{d} 26.0 \pm 0.29^{b-e}$ & $9.0 \pm 2.40^{\mathrm{ab}}$ & $99.0 \pm 1.00^{\mathrm{e}}$ & $125.9 \pm 6.47^{\text {b-e }}$ \\
\hline 6 & Hyb6 & $3.8 \pm 1.35^{\mathrm{b}-\mathrm{e}}$ & 100.4 & $10.39^{a-f}$ & 14 & $\mathrm{~d}_{25.8} \pm 0.35^{\mathrm{b}-\mathrm{e}}$ & $10.0 \pm 0.35^{\mathrm{ab}}$ & $99.0 \pm 1.00^{\mathrm{e}}$ & $125.9 \pm 5.31^{\mathrm{b}-\mathrm{e}}$ \\
\hline 7 & Hyb7 & $\pm 0.29^{\mathrm{bcd}}$ & 108.4 & $69.7 \pm 2.56^{\mathrm{c}-\mathrm{f}}$ & $133.7 \pm 0.61^{\mathrm{cd}}$ & $24.9 \pm 0.77^{\mathrm{e}-\mathrm{h}}$ & $10.0 \pm 1.10^{\mathrm{ab}}$ & $99.0 \pm 1.15^{\mathrm{e}}$ & $126.5 \pm 6.01^{b-e}$ \\
\hline 8 & Hyb8 & $41^{a b c}$ & & $46^{\mathrm{def}}$ & 140.0 & $.13^{\mathrm{e}-\mathrm{i}}$ & $11.0 \pm 1.56^{\mathrm{a}}$ & $99.0 \pm 1.00^{\mathrm{e}}$ & $.97^{\mathrm{bcc}}$ \\
\hline 9 & Hyb9 & $.46^{\mathrm{ab}}$ & 119.1 & $75.3 \pm 5.62^{\mathrm{a}-\mathrm{e}}$ & $155.0 \pm$ & $24.8 \pm 0.67^{\mathrm{e}-\mathrm{h}}$ & $11.0 \pm 0.20^{\mathrm{a}}$ & $98.0 \pm 1.15^{\mathrm{e}}$ & $124.5 \pm 5.02^{\mathrm{b}-\mathrm{g}}$ \\
\hline 10 & Hyb10 & bed & & b-f & $18^{\mathrm{cd}}$ & 24.4 & $12.0 \pm 0.99^{\mathrm{a}}$ & $98.0 \pm 1.00^{\mathrm{e}}$ & $7.95^{\mathrm{c}-\mathrm{h}}$ \\
\hline 11 & Hyb11 & $4.9 \pm$ & 127.6 & $75.3 \pm 5.70^{\mathrm{a}-\mathrm{e}}$ & $5.40^{\mathrm{a}-\mathrm{d}}$ & $24.4 \pm 0.68^{f-i}$ & $10.0 \pm 1.86^{\mathrm{ab}}$ & $100.0 \pm 2.00^{\mathrm{e}}$ & $125.4 \pm 4.52^{\mathrm{b}-\mathrm{f}}$ \\
\hline 12 & Hy & 4.0 & & & & & $0.80^{\mathrm{ab}}$ & $100.0 \pm 1.53^{\mathrm{e}}$ & $3.58^{\mathrm{b}-\mathrm{e}}$ \\
\hline 13 & Hyb13 & $4.6 \pm 0.19^{\mathrm{ab}}$ & 120.8 & $.84^{\mathrm{b}-\mathrm{f}}$ & $125.7 \pm 8.40^{\mathrm{d}}$ & $24.0 \pm 0.76^{\mathrm{hi}}$ & $11.0 \pm 0.90^{\mathrm{a}}$ & $99.0 \pm 1.53^{\mathrm{e}}$ & $117.7 \pm 4.61^{\mathrm{e}-\mathrm{i}}$ \\
\hline 14 & Hyb14 & $5.4 \pm 0.45^{\mathrm{a}}$ & 141.0 & $79.7 \pm 5.41^{\mathrm{abc}}$ & $150.0 \pm 17.12^{2}$ & $24.1 \pm 0.36^{\mathrm{ghi}}$ & $11.0 \pm 1.14^{\mathrm{a}}$ & $105.0 \pm 1.15^{\mathrm{d}}$ & $131.1 \pm 2.53^{b c}$ \\
\hline 15 & RD31 & $4.6 \pm 0.31^{\mathrm{ab}}$ & & $27^{\mathrm{a}-\mathrm{d}}$ & $139.7 \pm 8.46^{c}$ & 6 & 58 & 112.0 & $5.84^{\mathrm{a}} \mathrm{b}$ \\
\hline 16 & PSL2 & $3.7 \pm 0.12^{\mathrm{b}-\mathrm{e}}$ & & $73.7 \pm 5.81^{\mathrm{b}-\mathrm{f}}$ & $131.0 \pm 23.56^{\mathrm{cd}}$ & $27.5=$ & $10.0 \pm 0.40^{\mathrm{ab}}$ & $113.0 \pm 1.00^{\mathrm{b}}$ & $109.1 \pm 2.47$ \\
\hline 17 & PTT1 & & & & $142.0 \pm$ & & & $113.0 \pm 0.58^{\mathrm{b}}$ & 119.4 \\
\hline 18 & RD47 & $3.8 \pm 0.38^{\mathrm{b}-\mathrm{e}}$ & & $74.7 \pm 4.05^{\mathrm{a}-\mathrm{f}}$ & $171.0 \pm 14.87^{\mathrm{a}}$ & $26.2 \pm 0.18^{\mathrm{b}}$ & $10.0 \pm 0.61^{\mathrm{ab}}$ & $111.0 \pm 0.58^{\mathrm{c}}$ & $114.8 \pm 4.40^{h i}$ \\
\hline
\end{tabular}

Within each column, means followed by a common letter are not significantly different by the LSD test at the 5\% level of significance. Lot numbers 1-14 represent hybrids from crosses of TGMS lines with Thai cultivars (see Table 1). Lot numbers 15-18 represent high-yielding inbred Thai reference cultivars for comparison. Mean check is the mean grain yield of the four reference cultivars.

\section{Polymorphic markers and Positive alleles for agronomically desirable traits}

Among the hybrid lines tested, only Hyb1 showed $10 \%$ or greater yield mean check for all locations and years. Therefore, Hyb1 was selected for further study as it represents a consistently high-yielding cultivar. To identify polymorphic markers between the parents of Hyb1 and, at the same time, to identify positive alleles for agronomically desirable traits in each parent and the hybrid, eight functional markers controlling agronomically desirable traits were used to test pooled DNA of each parent and the hybrid. The results showed that four markers (TGW6, Ghd7, Badh2, and SSIIa) were polymorphic among the parents (Fig. 1). These markers, thus, 
Table 5 Inter-station yield evaluation of two-line hybrids planted at the Chainat Rice Research Center (CNrc) in 2016.

\begin{tabular}{|c|c|c|c|c|c|c|c|c|c|}
\hline No & List & $\begin{array}{c}\text { Gain yield } \\
\text { (t/ha) }\end{array}$ & $\begin{array}{l}\% \text { of Mean } \\
\text { check }(5.3)\end{array}$ & $\begin{array}{c}\text { Spikelet } \\
\text { fetility (\%) }\end{array}$ & $\begin{array}{l}\text { No. of seeds } \\
\text { per penicle }\end{array}$ & $\begin{array}{l}1000 \text { grain } \\
\text { weight }(g)\end{array}$ & $\begin{array}{l}\text { No. of } \\
\text { tillers }\end{array}$ & $\begin{array}{l}\text { Time to } \\
\text { flower (d) }\end{array}$ & $\begin{array}{l}\text { Height } \\
(\mathrm{cm})\end{array}$ \\
\hline 1 & Hyb1 & $6.4 \pm 0.68^{b c}$ & 120.0 & $72.0 \pm 2.10^{\mathrm{ab}}$ & $190.0 \pm 10.45^{\mathrm{a}-\mathrm{d}}$ & $27.0 \pm 2.10^{\mathrm{a}-}$ & $13.0 \pm 1.38^{\mathrm{a}}$ & $125.0 \pm 1.73^{\mathrm{a}}$ & $33.0 \pm 8.75^{\mathrm{abc}}$ \\
\hline 2 & Hyb2 & $5.6 \pm 1.23^{b c c}$ & 105.3 & $71.0 \pm 4.22^{\mathrm{ab}}$ & $192.0 \pm 32.38^{\mathrm{a}-\mathrm{d}}$ & $22.9 \pm 4.22^{f}$ & $13.0 \pm 0.61^{\mathrm{a}}$ & $105.0 \pm 1.73^{\text {cde }}$ & $106.0 \pm 3.99^{j \mathrm{k}}$ \\
\hline 3 & Hyb3 & $4.2 \pm 1.30^{\mathrm{e}}$ & 80.1 & $60.0 \pm 4.05^{c}$ & $197.0 \pm 9.67^{\mathrm{abc}}$ & $25.8 \pm 4.05^{\mathrm{cde}}$ & $15.0 \pm 0.56^{a}$ & $122.0 \pm 1.15^{\mathrm{ab}}$ & $122.0 \pm 4.72^{\mathrm{a}-\mathrm{d}}$ \\
\hline 4 & Hyb4 & $5.8 \pm 1.51^{\mathrm{bcc}}$ & 110.2 & $73.0 \pm 8.39^{\mathrm{ab}}$ & $175.0 \pm 34.77^{\mathrm{a}-\mathrm{e}}$ & $25.6 \pm 8.39^{\text {cde }}$ & $15.0 \pm 1.21^{\mathrm{a}}$ & $103.0 \pm 2.52^{\text {cde }}$ & $111.0 \pm 7.74^{\mathrm{ijk}}$ \\
\hline 5 & Hyb5 & $5.7 \pm 0.78^{\mathrm{bcc}}$ & 108.2 & $74.0 \pm 5.73^{\mathrm{ab}}$ & $168.0 \pm 13.70^{\mathrm{b}-\mathrm{e}}$ & $26.2 \pm 5.73^{\text {cde }}$ & $15.0 \pm 1.53^{\mathrm{a}}$ & $99.0 \pm 0.00^{\mathrm{e}}$ & $115.0 \pm 4.91^{\mathrm{ghi}}$ \\
\hline 6 & Hyb6 & $8.8 \pm 0.50^{\mathrm{a}}$ & 166.5 & $80.0 \pm 3.39^{a}$ & $184.0 \pm 0.85^{\mathrm{a}-\mathrm{e}}$ & $27.1 \pm 3.39^{\mathrm{a}-\mathrm{d}}$ & $15.0 \pm 0.26^{\mathrm{a}}$ & $108.0 \pm 2.52^{\mathrm{c}}$ & $122.0 \pm 3.91^{\mathrm{d}-\mathrm{g}}$ \\
\hline 7 & Hyb7 & $6.0 \pm 0.72^{b c c}$ & 112.8 & $71.0 \pm 7.32^{\mathrm{ab}}$ & $161.0 \pm 16.31^{\mathrm{de}}$ & $25.6 \pm 7.32^{\mathrm{cde}}$ & $17.0 \pm 2.05^{\mathrm{a}}$ & $102.0 \pm 3.79^{\text {de }}$ & $112.0 \pm 7.20^{\mathrm{h}-\mathrm{k}}$ \\
\hline 8 & Hyb8 & $6.0 \pm 0.97^{b c c}$ & 114.2 & $72.0 \pm 4.39^{\mathrm{ab}}$ & $173.0 \pm 8.86^{\mathrm{b}-\mathrm{e}}$ & $24.3 \pm 4.39^{\mathrm{ef}}$ & $15.0 \pm 2.34^{\mathrm{a}}$ & $101.0 \pm 2.52^{\mathrm{de}}$ & $116.0 \pm 2.00^{f-i}$ \\
\hline 9 & Hyb9 & $6.3 \pm 0.90^{b c}$ & 119.5 & $76.0 \pm 3.47^{\mathrm{ab}}$ & $201.0 \pm 7.99^{a b c}$ & $25.6 \pm 3.47^{\text {cde }}$ & $13.0 \pm 0.58^{a}$ & $106.0 \pm 4.62^{\mathrm{cd}}$ & $116.0 \pm 5.72^{\mathrm{f}-\mathrm{i}}$ \\
\hline 10 & Hyb10 & $5.9 \pm 1.13^{\mathrm{bcc}}$ & 110.5 & $75.0 \pm 7.37^{\mathrm{ab}}$ & $177.0 \pm 12.21^{\mathrm{a}-\mathrm{e}}$ & $25.2 \pm 7.37^{\text {cde }}$ & $15.0 \pm 0.51^{\mathrm{a}}$ & $103.0 \pm 2.52^{\mathrm{cde}}$ & $114.0 \pm 3.20^{g-j}$ \\
\hline 11 & Hyb11 & $5.7 \pm 0.71^{\mathrm{bcc}}$ & 107.6 & $78.0 \pm 6.25^{\mathrm{ab}}$ & $191.0 \pm 1.63^{\mathrm{a}-\mathrm{d}}$ & $24.8 \pm 6.25^{\mathrm{de}}$ & $14.0 \pm 1.21^{\mathrm{a}}$ & $121.0 \pm 6.00^{\mathrm{ab}}$ & $113.0 \pm 9.82^{\text {hij }}$ \\
\hline 12 & Hyb12 & $6.5 \pm 1.16^{\mathrm{bc}}$ & 122.1 & $75.0 \pm 4.07^{\mathrm{ab}}$ & $173.0 \pm 8.25^{\mathrm{b}-\mathrm{e}}$ & $25.7 \pm 4.07^{\mathrm{cd}}$ & $14.0 \pm 1.29^{\mathrm{a}}$ & $102.0 \pm 2.08^{\mathrm{de}}$ & $120.0 \pm 4.45^{\mathrm{e}-\mathrm{h}}$ \\
\hline 13 & Hyb13 & $6.5 \pm 1.29^{\mathrm{bc}}$ & 123.2 & $79.0 \pm 3.26^{\mathrm{a}}$ & $165.0 \pm 27.31 \mathrm{cde}$ & $25.7 \pm 3.26^{\mathrm{cde}}$ & $15.0 \pm 3.26^{\mathrm{a}}$ & $102.0 \pm 2.08^{\mathrm{de}}$ & $105.0 \pm 6.27^{\mathrm{k}}$ \\
\hline 14 & Hyb14 & $8.0 \pm$ & 151.8 & $79.0 \pm 2.57^{\mathrm{a}}$ & $193.0 \pm 12.53^{\mathrm{a}-\mathrm{d}}$ & $24.9 \pm 2.57^{\mathrm{c}-\mathrm{f}}$ & $15.0 \pm 3.12^{\mathrm{a}}$ & $104.0 \pm 0.00^{c c}$ & $128.0 \pm 3.70^{\mathrm{cde}}$ \\
\hline 15 & RD31 & $76^{\mathrm{cde}}$ & & $5.00^{\mathrm{ab}}$ & $182.0 \pm 4.58^{\mathrm{a}-\mathrm{e}}$ & $.00^{\mathrm{a}}$ & $82^{\mathrm{a}}$ & $89^{\mathrm{ab}}$ & $7^{\mathrm{a}-\mathrm{d}}$ \\
\hline 16 & PSL2 & $5.2 \pm 0.71^{\mathrm{cde}}$ & & $68.0 \pm 3.92^{b c}$ & $185.0 \pm 15.97^{\mathrm{a}-\mathrm{c}}$ & $28.9 \pm 3.92^{\mathrm{a}}$ & $13.0 \pm 2.30^{\mathrm{a}}$ & $120.0 \pm 1.73^{\mathrm{ab}}$ & $8.0 \pm 5.38^{\mathrm{ijk}}$ \\
\hline 17 & PTT1 & $4.8 \pm 0.64^{\mathrm{de}}$ & & $70.0 \pm 1.99^{\mathrm{ab}}$ & $154.0 \pm 4.02^{\mathrm{e}}$ & $26.3 \pm 1.99^{\mathrm{cde}}$ & $15.0 \pm 1.18^{\mathrm{a}}$ & $124.0 \pm 1.73^{\mathrm{a}}$ & $122.0 \pm 6.11^{\mathrm{d}-\mathrm{g}}$ \\
\hline 18 & RD47 & $5.8 \pm 1.16^{\mathrm{bcc}}$ & & $67.0 \pm 3.04^{b c}$ & $182.0 \pm 2.20^{\mathrm{a}-\mathrm{e}}$ & $28.6 \pm 3.04^{\mathrm{ab}}$ & $13.0 \pm 2.23^{\mathrm{a}}$ & $120.0 \pm 1.73^{\mathrm{ab}}$ & $112.0 \pm 8.99^{h-k}$ \\
\hline
\end{tabular}

Within each column, means followed by a common letter are not significantly different by the LSD test at the 5\% level of significance. Lot numbers 1-14 represent hybrids from crosses of TGMS lines with Thai cultivars (see Table 1). Lot numbers 15-18 represent high-yielding inbred Thai reference cultivars for comparison. Mean check is the mean grain yield of the four reference cultivars.

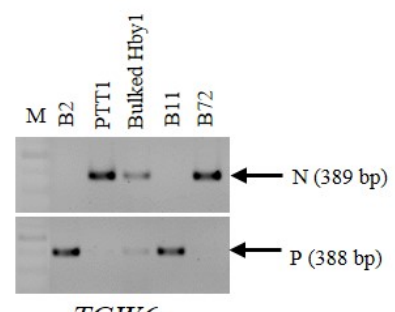

TGW6

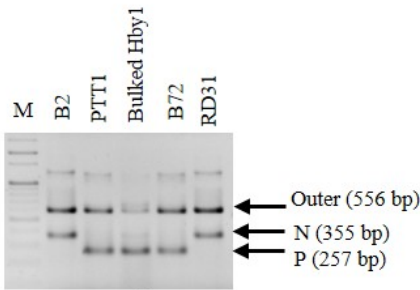

Badh2
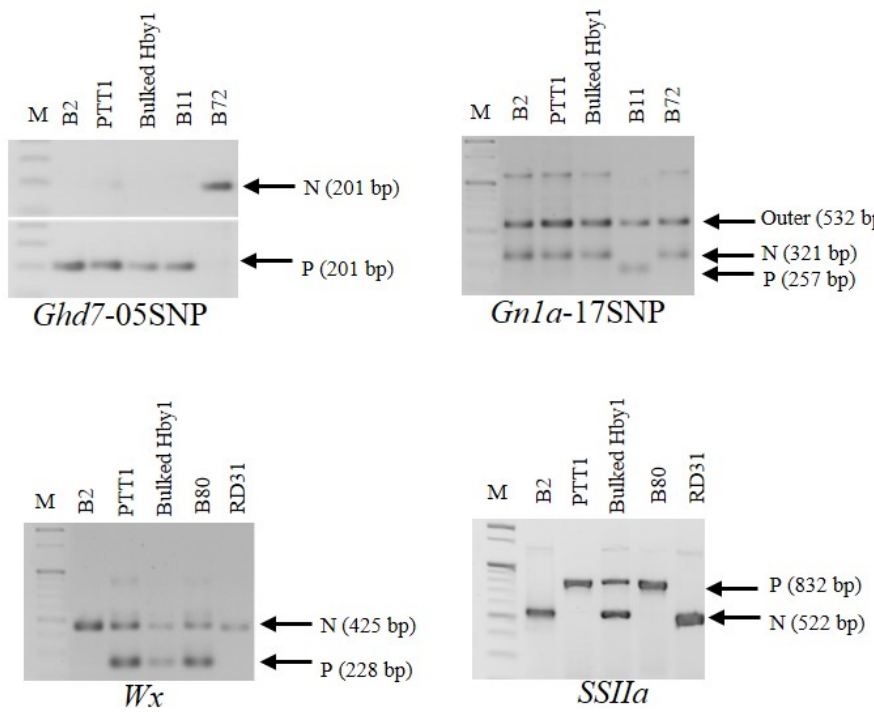

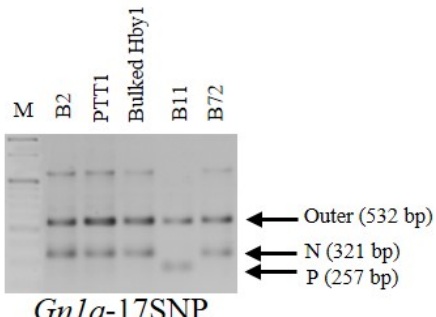

Gn1a-17SNP

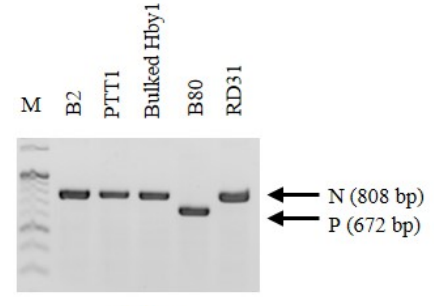

$S 5 n$

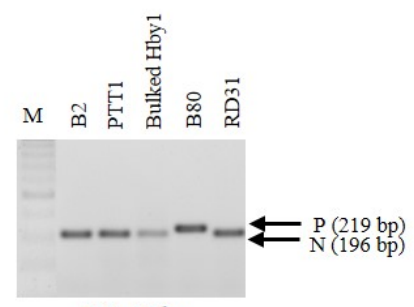

$W x-G l u$

Fig. 1 Polymorphic markers and positive alleles for agronomically desirable traits: $\mathrm{P}=$ positive allele; $\mathrm{N}=$ negative allele; $\mathrm{M}=$ marker; $\mathrm{B} 2$ = TGMS female parent; PTT1 = male parent; B11, B80, B72, and RD31 = negative and/or positive controls in the indicated markers. 


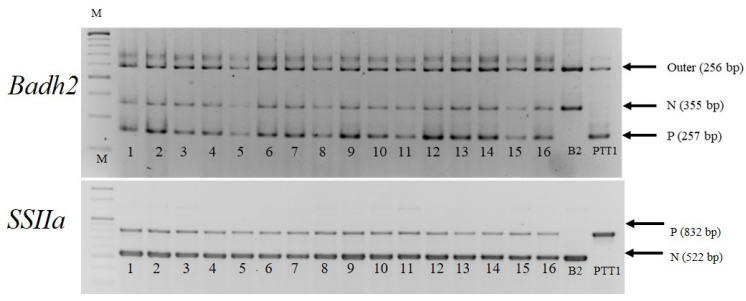

Fig. 2 Samples of seed purity test of Hyb1 using two functional markers controlling fragrance(Bahd2) and gelatinization temperature (SSIIa): $\mathrm{N}=$ negative allele; $\mathrm{P}=$ positive allele; $\mathrm{B} 2=$ TGMS female parent; PTT1 $=$ male parent; No. 1-16 = individual Hyb1 plants.

can be used to test seed purity of the hybrid. The maternal TGMS line (B2) has positive alleles for two yield-enhancing genes, TGW6 and Ghd7. The male parental line (PTT1) has positive alleles for Ghd7 controlling yield components, Badh2 affecting fragrance, $W x$ influencing amylose content, and SSIIa having major effect on gelatinization temperature. Fragrance, amylose content and gelatinization temperature are important attributes of rice eating and cooking quality. The results from genotyping with these markers were in agreement with their phenotypes.

\section{Seed purity test}

Seed contamination is a major problem for hybrid seed production. Therefore, purity assessment of hybrid seed lots is critical. Using two functional markers for fragrance (Bahd2) and gelatinization temperature (SSIIa), the seed purities of 30 random plants of Hyb1 were tested. The results showed that all the tested plants had alleles from the parents for both the tested markers, suggesting no contamination among Hyb1 seeds (Fig. 2).

\section{DISCUSSION}

Hybrid rice lines are alternative ways to increase yield in rice. In this study, 14 rice hybrids were generated, of which several showed higher yields than high-yielding inbred reference cultivars in some locations or growing seasons. However, only one hybrid, Hyb1, yielded 10\% more than the average yield of the reference cultivars at all tested years and locations. Moreover, Hyb1 showed 16 and 10\% higher yields than the average yield of the reference cultivars at PTrc in 2015 and 2016, respectively, and 37 and $20 \%$ higher yields than the average yield of the reference cultivars at BIOTEC in 2015 and at CNrc, in 2016, respectively. In comparison, the increase in yield for hybrid rice over inbred lines in other studies was less pronounced. For example, ordinary hybrid rice out-yielded the inbred rice by $4.53-6.17 \%$ and $4.35-4.69 \%$ at no added nitrogen (N0) and at $90 \mathrm{~kg} \mathrm{~N} / \mathrm{ha}$ (N90), respectively [30]; and in another study, early-season hybrids showed less than $5 \%$ greater yields over inbred lines [31]. Despite the increased yield over inbred reference cultivars, Hyb1 yields are lower than those reported in other studies [32-34]. One factor causing lower yields in our study could be the less amounts of fertilizer used in our study. In addition, our plant materials were different from other reports.

A plausible explanation for the higher yields of Hyb1 compared with reference cultivars could be the higher number of spikelets per panicle, which is reported to be a major factor determining yield [34-38]. However, in another report, hybrids with lower spikelet per panicle than inbreds showed greater yields [30]. Hyb1 also tended to have higher spikelet fertility than the inbred reference cultivars, similar to that reported by Huang et al [33]. However, these results were different from a previous report [34], probably because their hybrids were intersub-specific hybrids, whereas Hyb1 is an indicaindica hybrid.

Seed impurity in hybrids could be due to selfing of sterile lines, cross-pollination and mechanical mixture [12]. Male sterility of the maternal lines used in this study was controlled by temperature. Therefore, fluctuation of temperature could affect pollen sterility leading to selfing. In addition, crosspollination from other pollen shedders is not uncommon because male sterile maternal lines are ready to receive other pollens from nearby pollen shedders. Furthermore, mechanical mixture during processing is another factor that should be considered for the production of hybrid seeds. No contaminants were detected among Hyb1 seeds, suggesting that the male sterility of the maternal line was stable throughout the period of hybrid seed generation.

Several studies reported that the use of SSR markers to assess seed purity of parental lines and hybrids is superior over a grow-out test in terms of cost and time $[8,10,12]$. Single polymorphic SSR marker was proposed for routine purity analysis for commercial hybrid seeds [39]. However, using 2-3 SSR markers was demonstrated to be more superior over single marker analysis in accuracy detection of impurities in hybrids [10]. In this present study, two functional markers controlling fragrance (Bahd2) and gelatinization temperature (SSIIa) were used to assess the genetic purity of Hyb1 using normal 
agarose gel. The processes for using these markers were much easier and more friendly than those of SSR. The profiles of the parental lines were clearly distinguishable for these markers, which facilitated the identification of contaminant seeds. No contaminants in this hybrid seed lot were detected, which could explain the consistency of agronomic traits, in particular the markedly greater yield than that of its PTT1 male parent. This results could be one explanation for the high yield of this hybrid. In addition, the results from genotyping using other markers indicated that the parental lines contained several different positive alleles for agronomically desirable traits, including yield-enhancing genes and eating and cooking quality genes. The Hyb1 hybrid inherited all the positive alleles from its parents, supporting its high heterosis. The information obtained, including the knowledge, the hybrid and the F2 seeds from this hybrid, will be useful for future rice breeding in Thailand.

\section{Appendix A. Supplementary data}

Supplementary data associated with this article can be found at http://dx.doi.org/10.2306/ scienceasia1513-1874.2021.019.

Acknowledgements: We thank Dr. Kriangsak Suwantaradon, Dr. Ngamchuen Kongseree, and Suradet Palawisut for helpful suggestion with field experiments and related measurements. We acknowledge colleague and friends at Pathum Thani Rice Research Center and Chainat Rice Research Center for help with field experiments. We are grateful to Dr. Peera Jaruampornpan and Dr. Philip James Shaw for their critical reading and comments on the manuscript. This work was supported by Agricultural Research Development Agency (ARDA); and National Science and Technology Development Agency(NSTDA).

\section{REFERENCES}

1. USDA (2020) World Agricultural Production. Available at: https://apps.fas.usda.gov/psdonline/ circulars/production.pdf, pp 30.

2. Virmani SS (2003) Advances in hybrid rice research and development in the tropics. In: Virmani SS, Mao CX, Hardy B (eds) Proceedings of the 4th International Symposium on Hybrid Rice, Hanoi, Vietnam. pp 7-20.

3. Maruyama K, Araki H, Kato H (1991) Thermosensitive genic male sterility induced by irradiation. In: Rice Genetics II, International Rice Research Institute, Manila, pp 227-235.

4. Wang YG, Xing QH, Deng QY, Liang FS, Yuan LP, Weng ML, Wang B (2003) Fine mapping of the rice thermos-sensitive genic male-sterile gene tms5. Theor Appl Genet 107, 917-921.
5. Dong NV, Subudhi PK, Luong PN, Quang VD, Quy TD, Zheng HG, Wang B, Nguyen HT (2000) Molecular mapping of a rice gene conditioning thermosensitive genic male sterility using AFLP, RFLP, and SSR techniques. Theor Appl Genet 100, 727-734.

6. Reddy O, Siddiq E, Ali N, Hussain AJ, Nimmakayala P, Ramasamy P (2000) Genetic analysis of temperature-sensitive male sterility in rice. Theor Appl Genet 100, 794-801.

7. Mao CX, Virmani SS, Kumar I (1996) Technological innovations to lower the cost of hybrid rice seed production. In: Proceedings of Third International Symposium on Hybrid Rice, Directorate of Rice Research, Hyderabad, India. pp 111-128.

8. Bora A, Choudhury PR, Pande V, Mandal AB (2016) Assessment of genetic purity in rice (Oryza sativa L.) hybrids using microsatellite markers. 3 Biotech 6, 50.

9. Noli E, Conti S, Maccaferri M, Sanguineti MC (1999) Molecular characterization of tomoto cultivars. Seed Sci Technol 27, 1-10.

10. Sundaram RM, Naveenkumar B, Biradar SK, Balachandran SM, Mishra B, Ilyas Ahmed M, Viraktamath BC, Ramesha MS, et al (2008) Identification of informative SSR markers capable of distinguishing hybrid rice parental lines and their utilization in seed purity assessment. Euphytica 163, 215-224.

11. McCouch SR, Chen X, Panaud O, Temnykh S, Xu Y, Cho YG, Huang N, Ishii T, et al (1997) Microsatellite marker development, mapping and applications in rice genetics and breeding. Plant Mol Biol 35, 89-99.

12. Ye-yun X, Zhan Z, Yi-ping X, Long-ping Y (2005) Identification and purity test of super hybrid rice with SSR molecular markers. Rice Sci 12, 7-12.

13. Saxena RK, Saxena KB, Varshney RK (2010) Application of SSR markers for molecular characterization of hybrid parents and purity assessment of ICPH 2438 hybrid of pigeonpea (Cajanus cajan L. Millspaugh). Mol Breed 26, 371-380.

14. Pallavi HM, Gowda Rame, Shadakshari YG, Bhanuprakash K, Vishwanath K (2011) Identification of SSR markers for hybridity and seed genetic purity testing in sunflower (Helianthus annuus L.). Helia 34, 59-66.

15. Wu M, Jia X, Tian L, Lv B (2006) Rapid and reliable purity identification of F1 hybrids of maize (Zea may L.) using SSR markers. Mol Plant Breeding 3, 381-384.

16. Aly AA, Eliwa NE, Maraei RW (2019) Physiological and molecular studies on ISSR in two wheat cultivars after exposing to gamma radiation. ScienceAsia 45, 436-445.

17. Kumar MC, Vishwanath K, Shivakumar N, Rajendra PS, Radha BN (2012) Utilization of SSR markers for seed purity testing in popular rice hybrids (Oryza sativa L.). Ann Plant Sci 1, 1-5.

18. Andersen JR, Lübberstedt $\mathrm{T}$ (2003) Functional markers in plants. Trends Plant Sci 8, 554-560. 
19. Ingvardsen C, Schejbel B, Lübberstedt $T$ (2008) Functional markers in resistance breeding. In: Progress in Botany, Springer, Berlin, Heidelberg, pp 61-87.

20. Bao JS, Corke H, Sun M (2006) Nucleotide diversity in starch synthase IIa and validation of single nucleotide polymorphisms in relation to starch gelatinization temperature and other physicochemical properties in rice (Oryza sativa L.). Theor Appl Genet 113, 1171-1183.

21. Bradbury LMT, Henry RJ, Jin Q, Reinke RF, Waters DLE (2005) A perfect marker for fragrance genotyping in rice. Mol Breed 16, 279-283.

22. Ji Q, Lu J, Chao Q, Zhang Y, Zhang M, Gu M, Xu M (2010) Two sequence alterations, a 136 bp InDel and an A/C polymorphic site, in the $S 5$ locus are associated with spikelet fertility of indica-japonica hybrid in rice. J Genet Genomics 37, 57-68.

23. Jin L, Lu Y, Shao Y, Zhang G, Xiao P, Shen S, Corke $\mathrm{H}$, Bao J (2010) Molecular marker assisted selection for improvement of the eating, cooking and sensory quality of rice (Oryza sativa L.). J Cereal Sci 51, 159-164.

24. Gao L, Zhou M, Chen R, Gao H, Yan Q, Zhou W, Deng G (2012) Developing and validating the functional marker of rice Waxy gene. Rice Genomics Genet 3, 61-65.

25. Kim SR, Ramos J, Ashikari M, Virk PS, Torres EA, Nissila E, Hechanova SL, Mauleon R, et al (2016) Development and validation of allele-specific SNP/indel markers for eight yield-enhancing genes using whole-genome sequencing strategy to increase yield potential of rice, Oryza sativa L. Rice 9, ID 12.

26. Wanchana S, Toojinda T, Tragoonrung S, Vanavichit A (2003) Duplicated coding sequence in the waxy allele of tropical glutinous rice (Oryza sativa L.). Plant Sci 165, 1193-1199.

27. SAS Institute (2003) SAS Ver. 9.1.2 (C) 2002-2003, SAS Institute, Inc., Cary, NC.

28. Murray MG, Thompson WF (1980) Rapid isolation of high molecular weight plant DNA. Nucleic Acids Res 8, 4321-4325.

29. Pathaichindachote W, Panyawut N, Sikaewtung K, Patarapuwadol S, Muangprom A (2019) Assessment of the genetic diversity and indica allelic frequency of selected Thai upland landraces and rice varieties with special traits using SSR markers. Rice Sci 26, 393-403.

30. Huang L, Sun F, Yuan S, Peng S, Wang F (2018) Different mechanisms underlying the yield advantage of ordinary hybrid and super hybrid rice over inbred rice under low and moderate $\mathrm{N}$ input conditions. Field Crops Res 216, 150-157.

31. Chen JY, Du CX, Zhang HL, Dai DQ, Wu MY, Ma Y (2019) Limited yield advantage of early-season rice hybrids over inbreds in middle-lower reaches of the Yangtze River. Mol Breed 39, ID 107.

32. Shi W, Yin X, Struik PC, Xie F, Schmidt RC, Jagadish KS (2016) Grain yield and quality responses of tropical hybrid rice to high night-time temperature. Field Crops Res 190, 18-25.

33. Huang M, Zou Y, Jiang P, Xia B, Ibrahim M, He-jun A (2011) Relationship between grain yield and yield components in super hybrid rice. Agric Sci China 10, 1537-1544.

34. Wei Y, Zhang H, Blumwald E, Li H, Cheng J, Dai Q, Huo Z, Xu K, et al (2016) Different characteristics of high yield formation between inbred japonica super rice and inter-sub-specific hybrid super rice. Field Crops Res 198, 179-187.

35. Ying J, Peng S, He Q, Yang H, Yang C, Visperas RM, Cassman KG (1998) Comparison of high-yield rice in tropical and subtropical environments: I. Determinants of grain and dry matter yields. Field Crops Res 57, 71-84.

36. Sheehy JE, Dionora MJA, Mitchell PL (2001) Spikelet numbers, sink size and potential yield in rice. Field Crops Res 71, 77-85.

37. Ottis BV, Talbert RE (2005) Rice yield components as affected by cultivar and seedling rate. Agron $J$ 97, 1622-1625.

38. Katsura K, Maeda S, Horie T, Shiraiwa T (2007) Analysis of yield attributes and crop physiological traits of Liangyoupeijiu, a hybrid rice recently bred in China. Field Crops Res 103, 170-177.

39. Yashitola J, Thirumurugan T, Sundaram RM, Naseerullah MK, Ramesha MS, Sarma NP, Stone RV (2002) Assessment of purity of rice hybrids using microsatellite and STS markers. Crop Sci 42, 1369-1373. 


\section{Appendix A. Supplementary data}

Table S1 Details of the functional markers used in this study.

\begin{tabular}{|c|c|c|c|c|c|}
\hline No & Marker & Primer name & Primer sequence $\left(5^{\prime}-3^{\prime}\right)$ & $\operatorname{Tm}\left({ }^{\circ} \mathrm{C}\right)$ & Ref. \\
\hline \multirow[t]{2}{*}{1} & $S 5 n$ & $\mathrm{ADF}$ & GACCAGCACCATCTTCGATC & 61 & {$[22]$} \\
\hline & & $\mathrm{ADR}$ & CCTTGAGAGTTCACAGCGTG & & \\
\hline \multirow[t]{4}{*}{2} & badh2 & FragranceESP & TTGTTTGGAGCTTGCTGATG & 58 & {$[21]$} \\
\hline & & FragranceIFAP & CATAGGAGCAGCTGAAATATATACC & & \\
\hline & & FragranceINSP & CTGGTAAAAAGATTATGGCTTCA & & \\
\hline & & FragranceEAP & AGTGCTTTACAAAGTCCCGC & & \\
\hline \multirow[t]{3}{*}{3} & $W x$ & $\mathrm{WxF}$ & AGAGGGGGAGAGAGAGAaCG & 58 & [24] \\
\hline & & WxT & CAGGAAGAACATCTGCgAGT & & \\
\hline & & WxR & CСТAACCAAACATAACGAACG & & \\
\hline \multirow[t]{2}{*}{4} & $W x-G l u$ & Wx-Glu23F & TGCAGAGATCTTCCACAGCA & 60 & [26] \\
\hline & & Wx-Glu23R & GCTGGTCGTCACGCTGAG & & \\
\hline \multirow[t]{4}{*}{5} & SSIIa & SSIIaF22 & CAAGGAGAGCTGGAGGGGGC & 60 & {$[20]$} \\
\hline & & SSIIaNR1 & GGCCGTGCAGATCTTAACCAT & & \\
\hline & & SSIIaR21 & ACATGCCGCGCACCTGGAAA & & \\
\hline & & SSIIaf7 & CTGGATCACTTCAAGCTGTACGAC & & \\
\hline \multirow[t]{4}{*}{6} & Gn1a-17SNP & Gn1a-17SNP-OPF & TCGCAGGCACTGCACTTCA & 60 & {$[25]$} \\
\hline & & Gn1a-17SNP-OPR & GCCACCCTAGGTTTGATTCC & & \\
\hline & & Gn1a-17SNP-AF & CATACCTAGCGTTCTATGCTGA & & \\
\hline & & Gn1a-17SNP-GR & GGAAGATAAAGAAATTTCACATACC & & \\
\hline \multirow[t]{3}{*}{7} & Ghd7-05SNP & Ghd7-05SNP-F & TGCTTATGCGTACATCTGGAT & 58 & {$[25]$} \\
\hline & & Ghd7-05SNP-AR & TGGGTTCAAGCTCTCCACAT & & \\
\hline & & Ghd7-05SNP-TR & TGGGTTCAAGCTCTCCACAA & & \\
\hline \multirow[t]{3}{*}{8} & TGW6-1d & TGW6-1d-F & GCCAACTGATCAGACTGAG & 64 & {$[25]$} \\
\hline & & TGW6-1d-NR & CGTGGGGAGAGTCGATTCC & & \\
\hline & & TGW6-1d-PR & CGTGGGGAGAGTCGATTCG & & \\
\hline
\end{tabular}

